\title{
Article \\ One Row Keyboard: The Concept of Designing a Common Layout for Physical and Virtual Keyboards
}

\author{
Radosław Puka*D, Piotr Łebkowski and Jerzy Duda (D) \\ Faculty of Management, AGH University of Science and Technology, 30-059 Cracow, Poland; \\ plebkows@zarz.agh.edu.pl (P.Ł.); jduda@zarz.agh.edu.pl (J.D.) \\ * Correspondence: rpuka@zarz.agh.edu.pl
}

Citation: Puka, R.; Łebkowski, P.; Duda, J. One Row Keyboard: The Concept of Designing a Common Layout for Physical and Virtual Keyboards. Electronics 2021, 10, 663. https: / / doi.org/10.3390/ electronics10060663

Academic Editor: George

A. Tsihrintzis

Received: 3 February 2021

Accepted: 10 March 2021

Published: 12 March 2021

Publisher's Note: MDPI stays neutral with regard to jurisdictional claims in published maps and institutional affiliations.

Copyright: (c) 2021 by the authors. Licensee MDPI, Basel, Switzerland. This article is an open access article distributed under the terms and conditions of the Creative Commons Attribution (CC BY) license (https:/ / creativecommons.org/licenses/by/ $4.0 /)$.

\begin{abstract}
The development of technology brings the computerization of everyday life. The most common device used to communicate with a computer is the keyboard. Many papers have been devoted to the study of computer keyboards and virtual keyboards. The authors of this study propose a new concept of a single line keyboard named One Row Keyboard (ORK) which can be applied for both computer and virtual keyboards. Applying the proposed concept enables the number of keys on the keyboards to be reduced significantly. This allows for the development of keyboards even on really small mobile devices. The article also presents a new method of assigning characters to keys for the ORK and shows the use of this method to create exemplary ORK layouts. One Row Keyboard is also the first concept that makes it possible to create a fully alternating keyboard. Noteworthy is that the criterion of maximizing typing alternation is the most popular criterion for keyboard optimization used in the studies devoted to computer keyboards as it leads to an increase in typing speed and decrease in the number of errors.
\end{abstract}

Keywords: input device; one row keyboard; fully alternating keyboard; human-computer interaction

\section{Introduction}

Since Christopher Sholes patented the typewriter with the original QWERTY keyboard in 1878 [1], a number of keyboard designs of varying form and purpose have been developed. One of the most popular keyboards alternative to the computer QWERTY arrangement is the Dvorak keyboard [2]. In their research, Dvorak and Dealey presented a number of factors affecting the typing speed for a keyboard. They distinguished alternating typing as one of the most important factors. Research has continued on the impact of various factors affecting typing speed on a computer keyboard $[3,4]$ and the very keyboard arrangement problem is still the subject of scientific interest [5-7]. Anson et al. [8] proposed relocation of several letters to the numeric row. Smith et al. [9] presented the postulate that the physical shape of the keyboard should be modified. McLone et al. [10] proposed a curved keyboard, which is more ergonomic than the "standard" keyboard.

As technology advanced and the use of mobile devices became gradually more common, first keyboards dedicated to such devices were developed. The OPTI [11] and FITALY [12] keyboards deserve particular attention; they are equipped with four and two, respectively, space keys. The Metropolis [13] and ATOMIK [14] arrangements are other interesting projects, featuring hexagonal keys. Hsiao et al. [15] proposed a modification in QWERTY layout where all keys are arranged in one line. Bi et al. [16] proposed keyboard optimization for more than only one language. The papers devoted to the keyboard arrangement problem have more and more often concerned the issue of the development of keyboards for mobile devices $[17,18]$ and studying the effect of, inter alia, the size of the keys on the correctness of the text typed [19,20]. Chang et al. [21] studied the impact of the button position on muscle activity, touch time, and discomfort. Xiong and Muraki [22] suggested that tapping smaller buttons increase muscle effort. The single-row keyboard (named by the authors as 1Line keyboard), dedicated to mobile devices, was presented in [23]. This paper 
emphasized the importance of limiting the number of keys on virtual keyboards, necessary for their comfortable use. The article [24] proposed a virtual single-row keyboard that uses chordred methods for input character. In the article [25], an interesting proposal was presented to enter one character using a combination of two buttons (physical and virtual) on a mobile phone. The article [26] proposed a single-row keyboard, which can potentially be used both on a computer keyboard and on a mobile device. However, the proposed keyboard requires a dedicated device and special software to use it on a PC computer.

Problems of creating keyboard layouts both on virtual and computer keyboards, as well as adapting computer keyboard layouts to the needs of mobile devices, are problems still present and analyzed in the literature [26-28]. Due to the comfort of using one layout on both types of devices resulting, among others, from the lack of necessity to switch between different layouts, this problem seems to be very important. According to the authors, it can be decisive for the popularization of newly created layouts and strongly limits the popularity of layouts dedicated to only one type of device.

In the presented paper, the authors, when designing layouts (and assigning characters to keys), took into account the most important criteria for the optimization of computer keyboard layouts, including alternation of entering subsequent characters as well as the small size of keyboards in mobile devices. The proposed concept of designing One Row Keyboard (ORK) is the first concept that allows to create a fully alternating keyboard (for a computer keyboard). The significance of the hand alternation criterion was confirmed, among others, in papers $[6,29,30]$. In addition, due to the limited number of keys and the resulting possibility of increasing the size of the keys, ORK virtual keyboards seem to be more comfortable than keyboards imitating a full computer keyboard.

Section 2 describes the main assumptions of the proposed ORK keyboards and additionally proposes a method of creating the ORK keyboard design. Section 3 shows examples of ORK keypad groups. Discussion of the results is presented in Section 4. The last section summarizes the article.

\section{One Row Keyboard}

With the development of personal computers and their increasing use in everyday life, the problem of communication with a computer is gaining importance. The introduction of touch interfaces meant that the classical computer keyboard was replaced by a virtual keyboard, which is usually much smaller. Despite this, the QWERTY keyboard layout proposed in the 19th century is nowadays used in most mobile devices, even though they have much smaller sizes. This is related to the necessity to use many virtual keys, the small size of which may cause various difficulties in precise typing [22]. Thus, the main objective of this paper is to propose a concept of keyboard layout which can be used equally on a computer keyboard and on mobile devices, and at the same time will be adapted to the specifics of both types of devices. In the proposed solution, due to the small size of mobile devices, the authors focused on reducing the number of keys on the keyboard. Reduction in the number of keys is possible using the dead key functionality. Dead keys allow to modify the character assigned to the key struck immediately after the dead key has been pressed. This reduction was primarily aimed at the possibility of increasing the size of the keys on virtual keyboards used in mobile devices. When considering the computer keyboard, the authors took into account one of the most popular optimization criteria used in most of the studies devoted to the topic of keyboard layout optimization, i.e., typing alternation. As a limitation, the authors adopted the elimination of vertical movements of fingers while typing. This reduces fatigue in the hands caused by the movement of fingers. It could also reduce the number of typing errors resulting from striking a wrong key by a hand moving over the keyboard. 


\subsection{Definition of ORK Keyboard}

A keyboard satisfying the above assumptions is the keyboard proposed by the authors-the One Row Keyboard (ORK). A keyboard can be classified as an ORK keyboard if it satisfies the following condition:

Definition 1: [All letter keys are located in one row] and [they are operated by all fingers except thumbs or operated only by thumbs].

This definition distinguishes between two types of keyboards:

- keyboards supporting typing with all fingers,

- keyboards adapted to typing only with thumbs (or any other pair of fingers).

The above differentiation is based on the size of the keyboards used. Some keyboards, such as those in mobile phones, are generally too small to allow comfortable handling when using all fingers. Figure 1 presents an example of an ORK type keyboard layout.

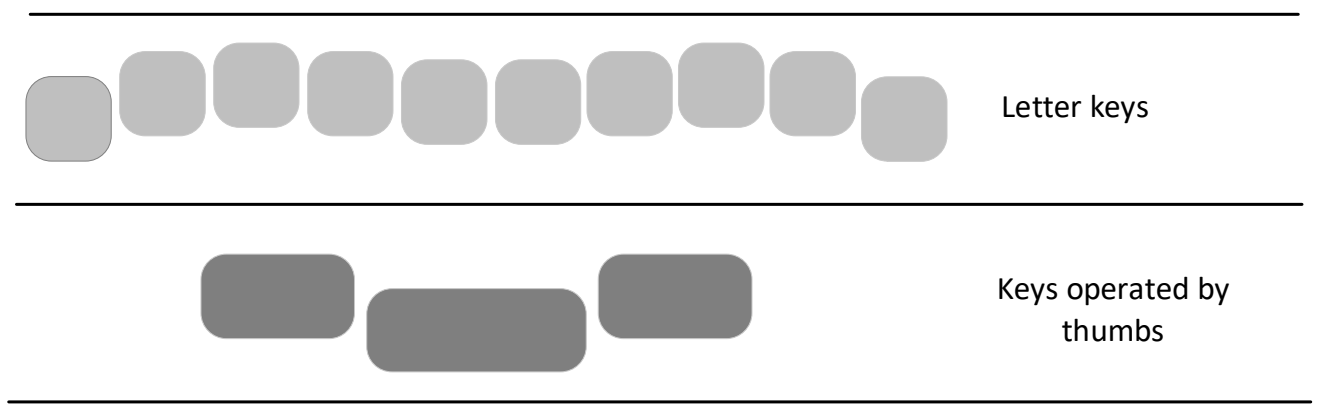

Figure 1. Example of One Row Keyboard (ORK) keyboard layout.

The example is a general one and presents the two types of ORK keyboards described. In the case of a thumb-only keyboard, the letter keys would be operated only with thumbs.

An enhancement of the ORK-type keyboard is the ORK+ type keyboard which must satisfy the following additional condition:

Definition 2: A keyboard has to be implementable on standard computer keyboards.

Thus, an ORK+ keyboard must simultaneously meet both: condition for the ORK keyboard and condition for the ORK+ keyboard.

The ORK + type has been proposed in order to enable the development of a keyboard which could be used for both mobile as well as standard PC keyboards (e.g., compliant with the ISO/IEC 9995-2:2009 standard [31]).

An example of an ORK + type keyboard arrangement with 10 letter keys is shown in Figure 2. The assignment of keys to fingers in the presented arrangement is the same as in touch typing on a similar keyboard [4].

In order to improve the ergonomics of typing, the authors propose an alternative to the illustrated arrangement of the characters of the ten-key ORK+ arrangement. The operation of the letter keys in the arrangement presented in Figure 3 is the same as in touch typing.

The ORK + arrangements proposed below is shown on an alternative version of a keyboard arrangement (Figure 3). The presented version of the keyboard layout allows to increase the maximum number of ORK+ keys to twelve. The arrangement shown in Figure 2 limits the maximum number of keys for the ORK+ layout to eleven. In this paper, the authors will focus only on the keys forming the ORK+; therefore, only those keys will be shown in illustrations presenting subsequent arrangements. In the case of the arrangements with 11 letter keys, the keyboard will be extended by one key, located directly to the right side of the key operated by the right-hand little finger. In order to be able to create an ORK + 
keyboard with 12 keys, it is necessary to shift (in relation to the one shown in Figure 3) the keys operated by the fingers of the left hand by one to the right. In this case, the little finger of the left hand will be responsible for operating the two keys. An exemplary keyboard arrangement supporting characters available through the use of dead keys is presented as shown in Figure 4. The location and number of dead keys vary from one to another keyboard arrangement.

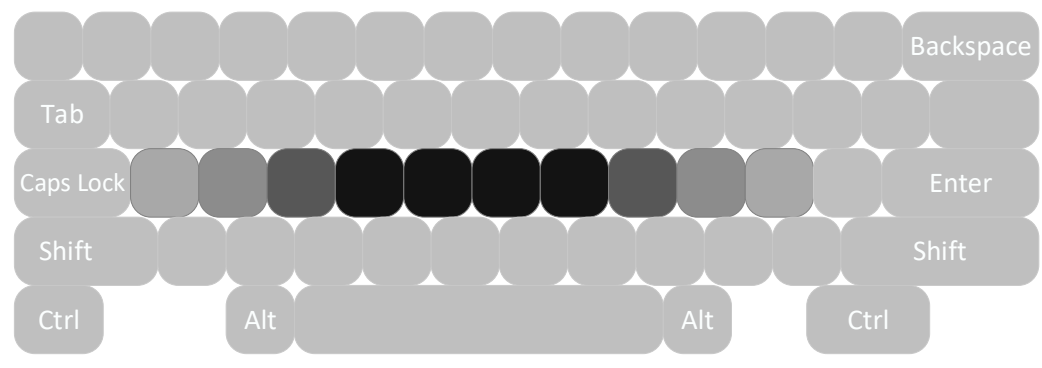

Key handling:

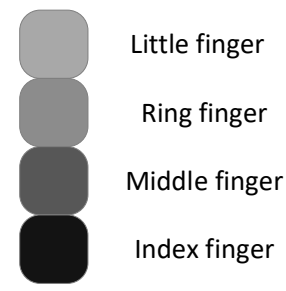

Figure 2. Example of keyboard arrangement of ORK+ type with 10 letter keys.

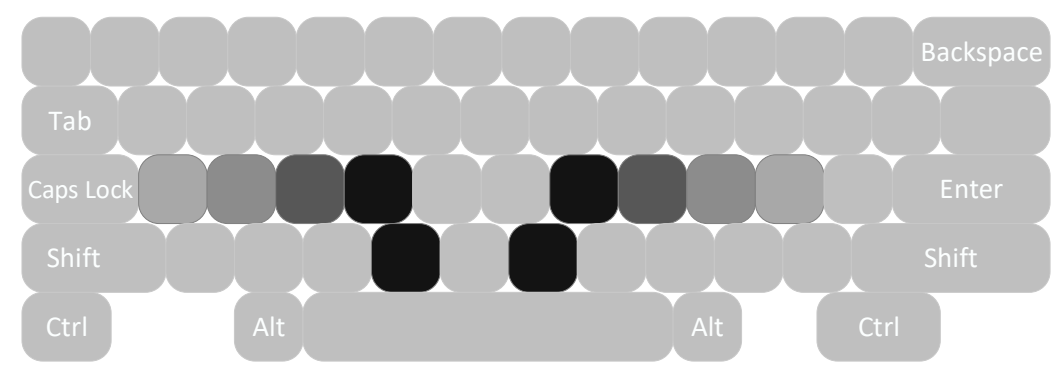

Figure 3. Alternative keyboard arrangement of ORK+ type with 10 keys.
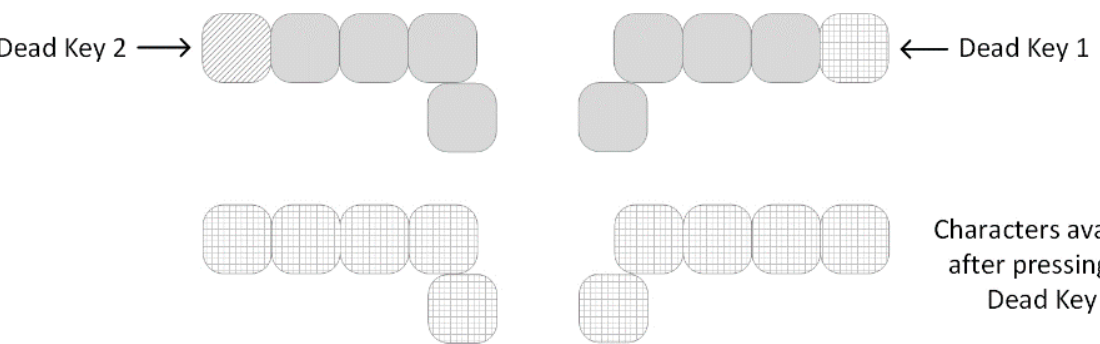

Characters available after pressing the Dead Key 1
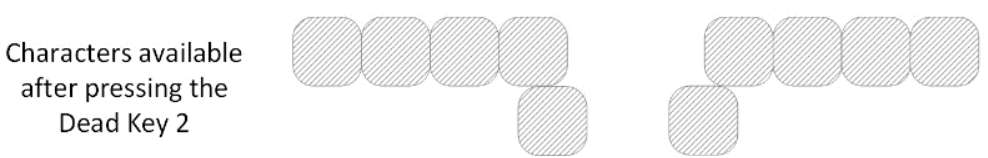

Figure 4. Schematic presentation of keyboards with two dead keys. 


\subsection{Method of Developing ORK Keyboard}

Designation of the ORK keyboard layout should be carried out in accordance with the following steps:

1. Determine the number of characters to be placed on the keyboard and the occurrence frequency of selected characters and their two-element combinations (bigrams).

2. Define the number of layout keys.

3. Determine the number and position of dead keys.

4. Select optimization criteria and their weights.

5. Assign characters to keys.

The first step in building an ORK keyboard is to determine the number of characters to be placed on the keyboard. This number is strongly dependent, e.g., on the language for which the layout is designed and the number of letters present in it. Additionally, the number of characters to be arranged on the keyboard may be increased by extending the character set by e.g., numerical characters. In order to evaluate a given layout, frequency occurrence statistics of each character considered and their two-element combinations (bigrams) are needed.

The next step is to determine the number of keys that will be used on the keyboard. Based on the definition 1, the number of keys cannot be smaller than 8 . At the same time, for ORK+ keyboards, the number of keys cannot exceed 12.

The next step is to determine the number of dead keys. Assuming $N$ is the number of keys in the layout, each dead key increments that number by N. Due to a limited number of keys, all keyboards use dead keys to access subsequent letters of the alphabet. Due to the nature of dead keys, for characters assigned to them to be typed (after a single keystroke), the next key needs to be a space. For example, the decimal point can be assigned to a dead key. In order to enter the decimal point, one needs to strike the dead key and then the space. The decimal point resulting will be with a space right after it. In this way, the result will be a character assigned to the dead key and a space. Therefore, the characters assigned to dead keys should be those which are usually directly followed by a space. The characters that do not require access through the use of dead keys will be called default characters. The number of dead keys depends on both the number of characters to be placed on the keyboard and on the number of keyboard keys. The maximum number of characters for a keyboard with $N$ keys is $N^{2}+N$, assuming each key is a dead key. This limitation should be taken into account when specifying the number of keys and the number of dead keys, with the assumed number of characters to be placed on the keyboard.

The fourth step is to establish a list of criteria to be considered during the optimization process. In the examples presented in the following section, the authors used selected criteria of computer keyboard optimization provided in the study [2]. According to the authors, the most important and most frequently appearing criterion of keyboard optimization in the literature is the alternation of typing, hence this criterion was used to optimize all proposed layouts. Another criterion used is the even use of each finger when typing. It should also be noted that some of the criteria in the literature related to e.g., minimizing the use of the bottom row and keyboard top row are fully realized by the ORK layout, since these rows are not used to input characters.

The last step of the method is to arrange characters on the keyboard, taking into account the adopted restrictions. Depending on the chosen optimization criteria, this task can be performed using heuristic algorithms (e.g., hill climbing, tabu search, simulated annealing, genetic algorithms) or manually. Manual characters setting may be justified when the only criterion is to map another layout as accurately as possible and transform it into an ORK layout. A mixed approach is also possible-some characters (especially non-letter characters) are set manually, while others are optimized.

The presented method of creating ORK keyboards was used to create several types of keyboards dedicated to the English language. In the next section, few examples of ORK + type keyboards are proposed. 


\section{ORK Type Keyboards}

In this part of the study, the authors proposed some examples of ORK type keyboards. The proposed keyboards have been divided into groups, and each group will be presented in the following subsections. The keyboards presented below were tested using the Microsoft Keyboard Layout Creator [32]. Thus, the use of ORK layouts does not require additional software or dedicated drivers. The occurrence frequency statistics of the letters and bigrams in English were used to arrange letters on the keyboards [33]. Each arrangement will be named according to the following pattern: the name of the arrangement-the name of the group of arrangements-the type of the arrangement (ORK or ORK+). All arrangements were developed using the random-restart iterative hill climbing algorithm.

\subsection{VX-ORK+ Arrangement Group}

The key feature of the VX-ORK+ group keyboard is as follows: ten letter keys, two of which are dead keys.

The first proposed VX-ORK + type keyboard is one of the possible QWERTY representations. The A-VX-ORK+ keyboard was developed by assigning the middle QWERTY row to the default characters. There are dead keys located in the locations of letters " $\mathrm{F}$ " and "J", with the full stop "." and comma "," characters assigned, respectively. The letters " $\mathrm{F}$ " and "J" are assigned to the full stop and comma keys on the QWERTY keyboard. The A-VX-ORK+ arrangement is presented in Figure 5.

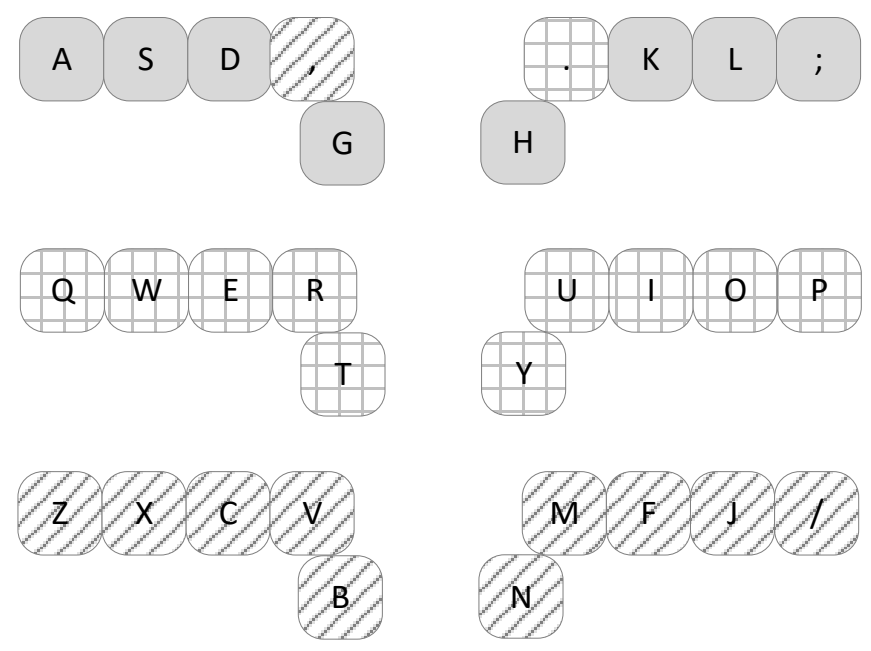

Figure 5. A-VX-ORK+ arrangement.

Dead keys are assigned to the keys operated by the index fingers due to the low frequency of occurrence of the letters " $\mathrm{F}$ " and " $\mathrm{J}$ " assigned to these keys in the QWERTY arrangement. Since the authors' objective is to arrange the dead keys symmetrically, the pair of letters was selected with the lowest total frequency. It is important, because all of the characters, except the default ones, need two keys to be typed (a dead key + the character key).

For this reason, the most frequent characters should be selected as defaults. The presented assignment of the QWERTY arrangement row to the specified dead key aims at increasing the usage frequency of the fingers of both hands during typing (a finger of one hand strikes a dead key, a finger of the other is responsible for typing the character).

The second proposed VX-ORK + type keyboard (Figure 6) is also the QWERTY keyboard adaptation. The default keys of the Q-VX-ORK+ keyboard form the first row of the QWERTY keyboard. Due to the assignment of dead keys to " $\mathrm{Q}$ " and " $\mathrm{P}$ " keys on the QWERTY keyboard, the letters " $Q$ " and " $\mathrm{P}$ " are assigned to the "," and "." keys on the QWERTY keyboard. 


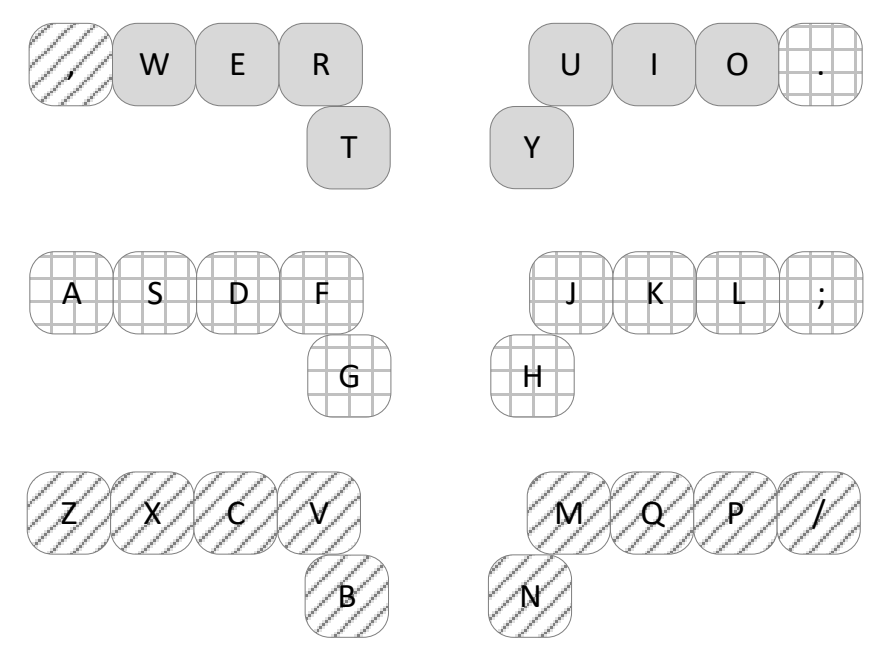

Figure 6. Q-VX-ORK+ arrangement.

The default characters of the Q-VX-ORK+ arrangement are in the first row of the QWERTY keyboard, due to the high prevalence of the letters. Dead keys are located on the keys operated by little fingers, because of the low prevalence of the letters " $\mathrm{Q}^{\prime}$ and " $\mathrm{P}$ ", which are moved to make space for the dead keys. The location of the dead keys is also based on the research by Dvorak and Dealey [2], according to which typing in the direction from the little finger to the index finger is more convenient than in the opposite direction. The assignment of the rows to the dead keys was chosen so as to maximize the total prevalence of the letters that are typed using fingers of both hands.

The last of the proposed keyboards, the VX-ORK+ type one, is the P-VX-ORK+ arrangement. The keyboard has been developed on the basis of the results of calculations for the following function:

$$
\text { F-VX-ORK }+: \max \left(\sum_{l \in \text { letters }} x_{l} * w_{l}+\sum_{b \in \text { bigrams }} y_{b} * w_{b}\right),
$$

where letters denote the set of all of the letters of the English alphabet, bigrams denote the set of all bigrams, $w_{l}$ and $w_{b}$ denote the frequency of a given letter and given bigram, respectively, while $x_{l}$ and $y_{b}$ are binary variables defined as follows:

$$
\begin{gathered}
x_{l}=\left\{\begin{array}{ll}
0 & \text { if theletter is not a default character } \\
1 & \text { if the letter is a default character }
\end{array},\right. \\
y_{b}=\left\{\begin{array}{ll}
0 & \text { if the bigram is typed with fingers of one hand } \\
1 & \text { if the bigram is typed with fingers of both hands }
\end{array} .\right.
\end{gathered}
$$

In the case of bigrams, it was only verified whether there is a switch of the hands between the first and the second letter typed. No account was taken of whether the letter not being a default character is typed with the fingers of the same hand (such cases were taken into account in the first component of the sum constituting the F-VX-ORK+ function). The objective behind including this component in the F-VX-ORK+ function (1) was to maximize alternating typing. According to certain research [2,5], alternating typing is one of the key factors that increases the typing speed and decreases the number of errors.

Based on the function presented, six-letter sets were developed-three for each corresponding hand:

- a set of letters assigned to default characters,

- $\quad$ a set of letters using dead keys which need to be typed with both hands (the dead key is operated by a different hand than the character to be typed),

- a set of letters using dead keys for which both the dead key and the letter key are operated with fingers of the same hand. 
Based on the distribution obtained, the letters are assigned to the keys according to the following criteria:

- maximizing the even distribution of typing load on individual fingers,

- minimizing the usage frequency for a single finger operating two consecutive keys.

The calculations resulted in the keyboard arrangement presented in Figure 7.

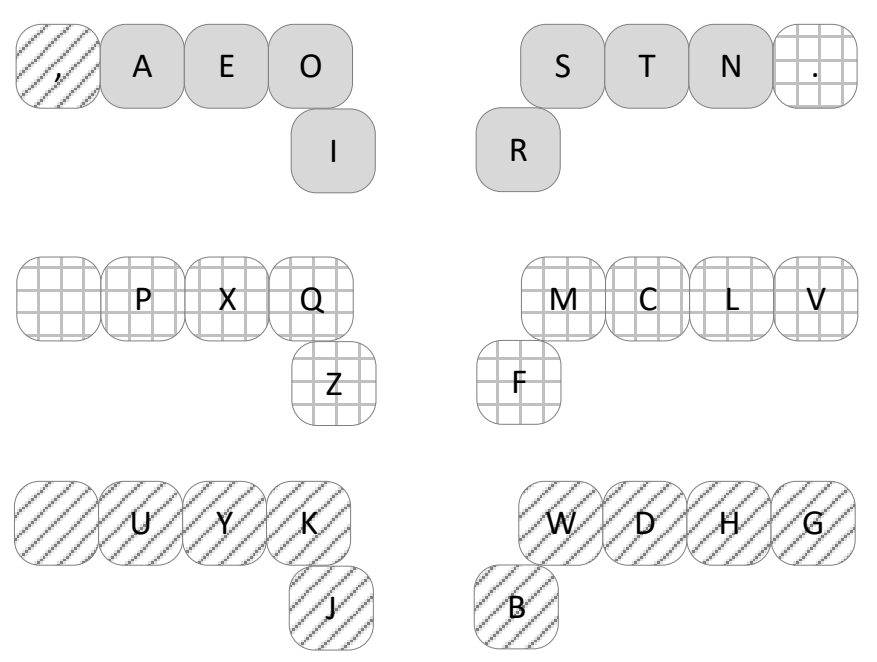

Figure 7. P-VX-ORK+ arrangement.

The keyboards of the VX-ORK+ group allow for the typing of up to 30 characters, including 28 letters (due to the location of the two dead keys). In the authors' opinion, VX-ORK + type keyboards are best characterized by the following two values: the frequency of typing bigrams with fingers of both hands (BI), and the prevalence of letters assigned to the default characters (DK- default key). The higher the DK value, the lower the value Keystrokes per Character (KSPC) [34]. For all ORK keyboards, the KSPC value is in the range [1;2]. The first value measures the presence of alternating typing and it should be maximized to the fully alternating typing. The second value measures the frequency of letters typed with just one strike, without using a dead key; also here: the higher the value, the better the arrangement. Table 1 presents the values of both metrics for the VX-ORK+ group arrangements.

Table 1. Assessment of VX-ORK+ group arrangements.

\begin{tabular}{cccc}
\hline & ASD & Q & P \\
\hline BI & $56.84 \%$ & $53.13 \%$ & $76.23 \%$ \\
DK & $29.90 \%$ & $49.33 \%$ & $65.04 \%$ \\
\hline
\end{tabular}

The results presented reveal that, against the metric proposed by the authors, by far the best of the arrangements analyzed is P-VX-ORK + , for which:

- more than three-quarters of the bigrams are typed alternatingly,

- the letters assigned to default characters account for nearly two thirds of all of the letters typed; only slightly more than one-third of the characters typed require the use of a dead key, that is, the use of two keys to type a single character.

The other two arrangements' alternating ratios (BI) are close to each other. A clear difference is noticeable for the DK metric; the value obtained for the Q-VX-ORK+ arrangement is definitely better.

\subsection{W-ORK+ Arrangement Group}

The keyboards of the W-ORK+ group have the following structure: eleven letter keys, including ten dead keys. 
The W-ORK+ keyboards proposed by the authors achieve a very high alternating ratio (BI), up to $100 \%$, which is possible thanks to the use of numerous (in the cases presented, at least ten) dead keys, and to assigning a given character to two keys on the keyboard, so that a given character may be typed (preceded by a dead key) with any of the hands. The example of an ABC-W-ORK+ arrangement presented here is based on the alphabetical order and comprises eleven letter keys (including ten dead keys); it is shown in Figure 8.
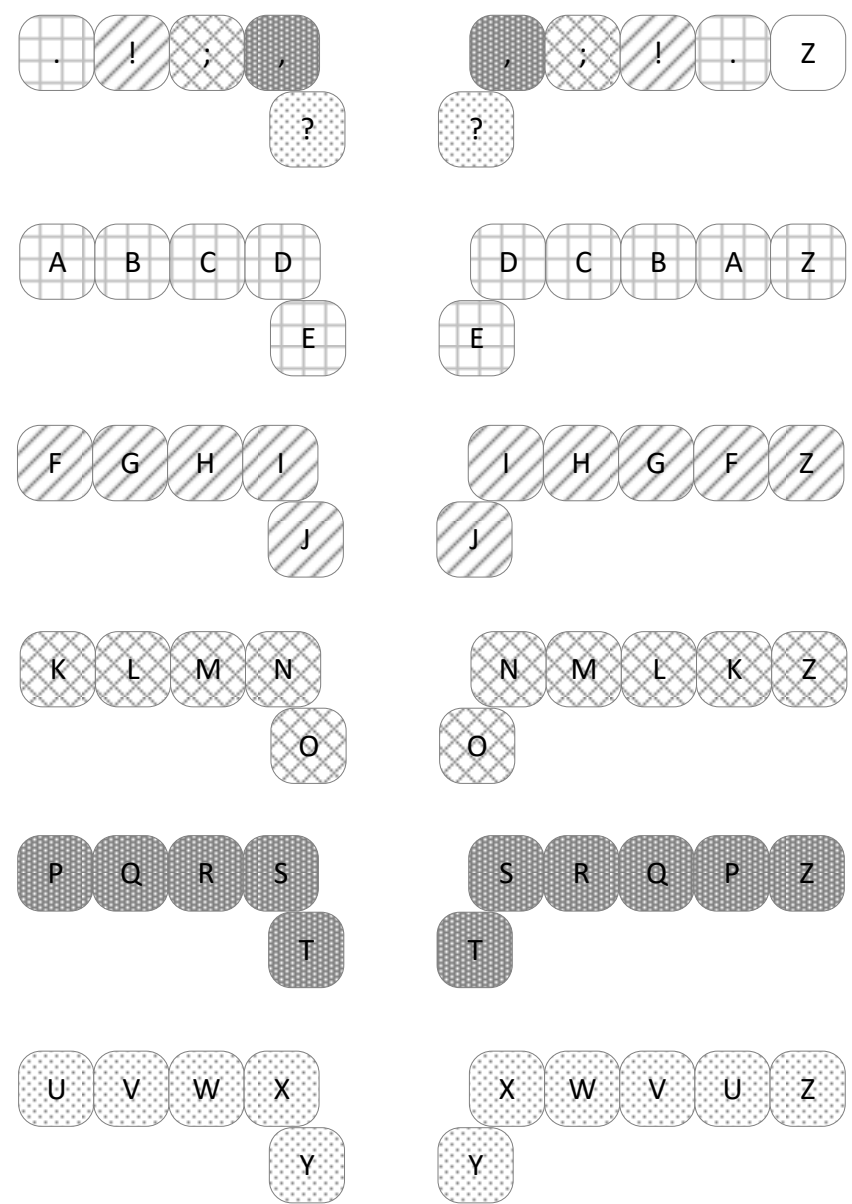

Figure 8. ABC-W-ORK+ arrangement.

The example of the ABC-W-ORK+ arrangement shows what the authors believes to be the key feature of the proposed W-ORK+ arrangement group: both all the character keys (save the letter " $Z$ " character) and the dead keys are arranged so that half of the arrangement (let us, say, to the left of the vertical axis) is as a mirror image of the other half. Therefore, each letter (with the exception of " $Z$ "), can be typed by any of the hands. Such arrangement has the following property: any specific character is assigned to the same finger, whichever of the two hands is typing it.

In the $A B C-W-O R K+$ arrangements, all the letters (except for the letter " $Z$ ") are assigned to dead keys. The objective of this solution is to maximize alternating ratio (BI), which is equal to $100 \%$ in the example described.

The high BI value was obtained at the expense of DK, which for the ABC-W-ORK+ arrangement is slightly over 0 (only the letter " $Z$ " is assigned to default character, while its prevalence is approximately $0.1 \%$ ). This means that typing of practically every letter requires the use of two keys.

Another arrangement presented is the Q-W-ORK+ arrangement. The arrangement is developed based on the QWERTY arrangement. All letters, except for " $\mathrm{N}$ " and " $\mathrm{M}$ ", are arranged in a manner similar to the QWERTY arrangement. For the Q-W-ORK+ 
arrangement, the BI ratio is $100 \%$. For comparison, for the standard QWERTY, BI is merely $52.87 \%$. The Q-W-ORK+ arrangement is shown in Figure 9.

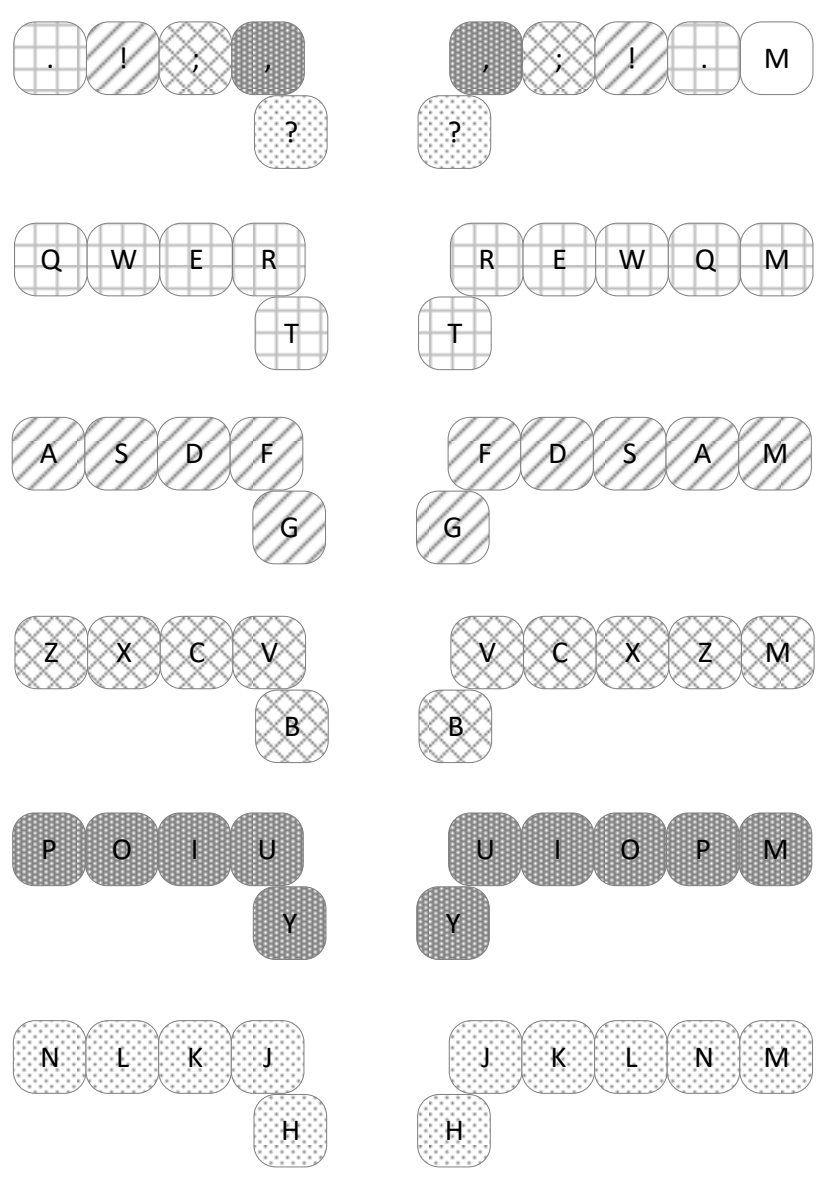

Figure 9. Q-W-ORK+ arrangement.

In the W-ORK+ arrangements presented here, the arrangement of letters is not optimized. The arrangements presented herein below are optimized against the following function:

$$
\text { F-W-ORK }+: \min \left(\sum_{f \in \text { fingers }}\left|\left(\sum_{l \in \text { letters }} x_{f l} * w_{l}\right)-o\right|+\sum_{l \in \text { letters }} z_{l} * w_{l}\right),
$$

where letters denotes the set of all letters in the English alphabet, fingers denotes the set of fingers of one hand, excluding the thumb (the assignment of characters to both hands is identical), $w_{l}$ denotes the given letter's prevalence, the $o$ parameter is the expected average value of the load to each of the fingers, while $x_{f l}$ and $z_{l}$ are binary variables defined as follows:

$$
x_{f l}=\left\{\begin{array}{l}
0 \quad \text { if the letter } l \text { is not typed with finger } f \\
1 \text { if the letter } l \text { is typed with finger } f
\end{array},\right.
$$

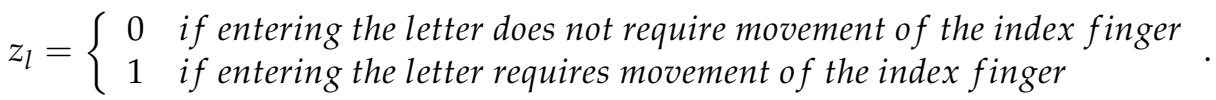

The value of the parameter $\mathrm{o}$ is calculated using the following formula:

$$
o=2 \times\left(100 \%-w_{d}\right) / 4,
$$

where $w_{d}$ is the frequency of the default character on a W-ORK+ keyboard.

The parameter $o$ is used to determine what should be the frequency of the letters typed with a given finger (four fingers of the hand are used for typing), assuming that every letter, except the letter assigned to the default character, requires two fingers (striking two keys). 
The load to the little finger of the right hand associated with typing the default character is neglected.

Optimization against the function defined above is designed to ensure even distribution of loads to all of the typing fingers (the first addend), as well as to minimize the frequency of the index finger movements (the second addend). The function omits the default character selected arbitrarily.

Based on the presented function (F-W-ORK + (4)), the My-W-ORK+ and U-W-ORK+ keyboards were developed. The main difference between them is in the letter assigned to the default character. For My-W-ORK+ arrangement, it is the letter " $\mathrm{Z}$ ", while for the U-W-ORK+ arrangement, it is the letter " $\mathrm{E}$ ". The other differences result from this assignment because the change in the default character changes the input data for the optimizing function.

Figure 10 presents the My-W-ORK+ arrangement.
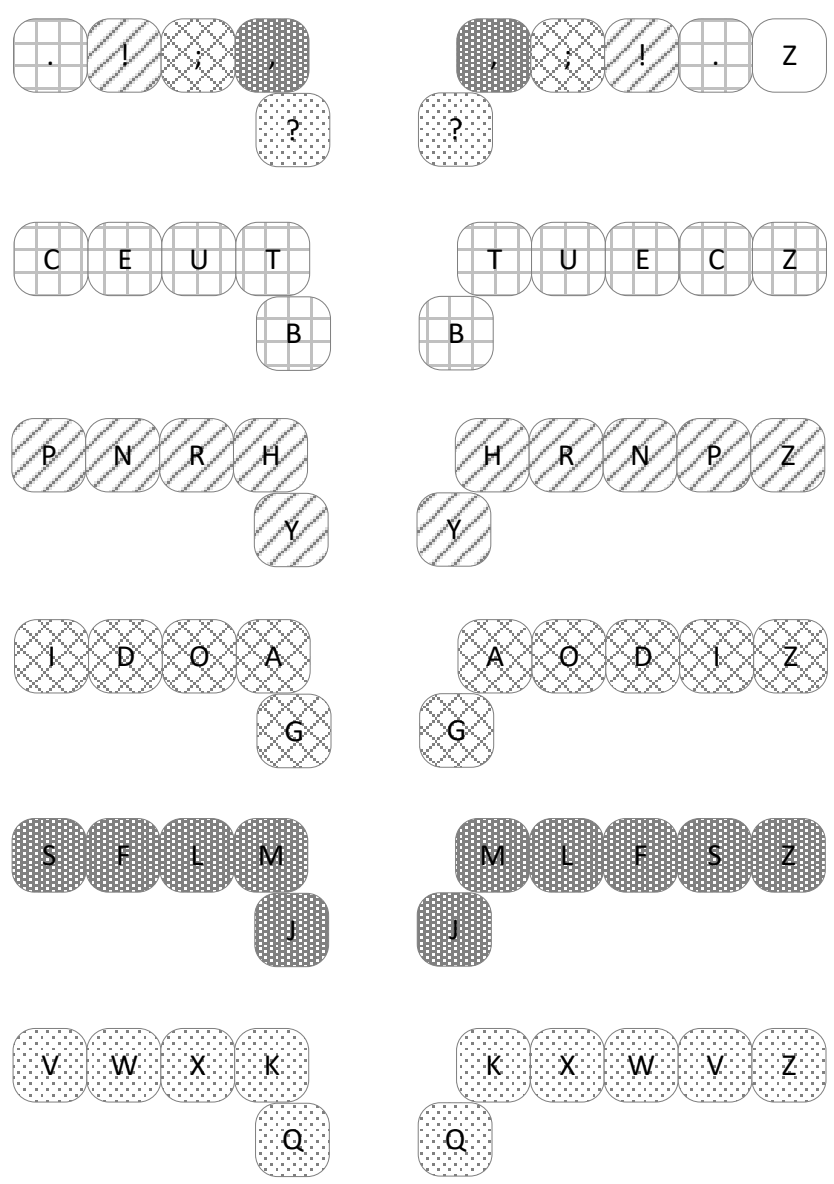

Figure 10. My-W-ORK+ arrangement.

The My-W-ORK+ arrangement is characterized by a load practically evenly distributed among all typing fingers. The difference between the most and least loaded fingers is $0.1 \%$. Statistically, the My-W-ORK+ arrangement requires the index finger movement only for one in twenty-two clicks.

Another presented arrangement is the W-U-ORK+ one. Its characteristic feature is the assignment of letter " $\mathrm{E}$ " to the default character. This allowed to obtain the DK ratio of $12.5 \%$. The U-W-ORK+ arrangement is presented in Figure 11. 


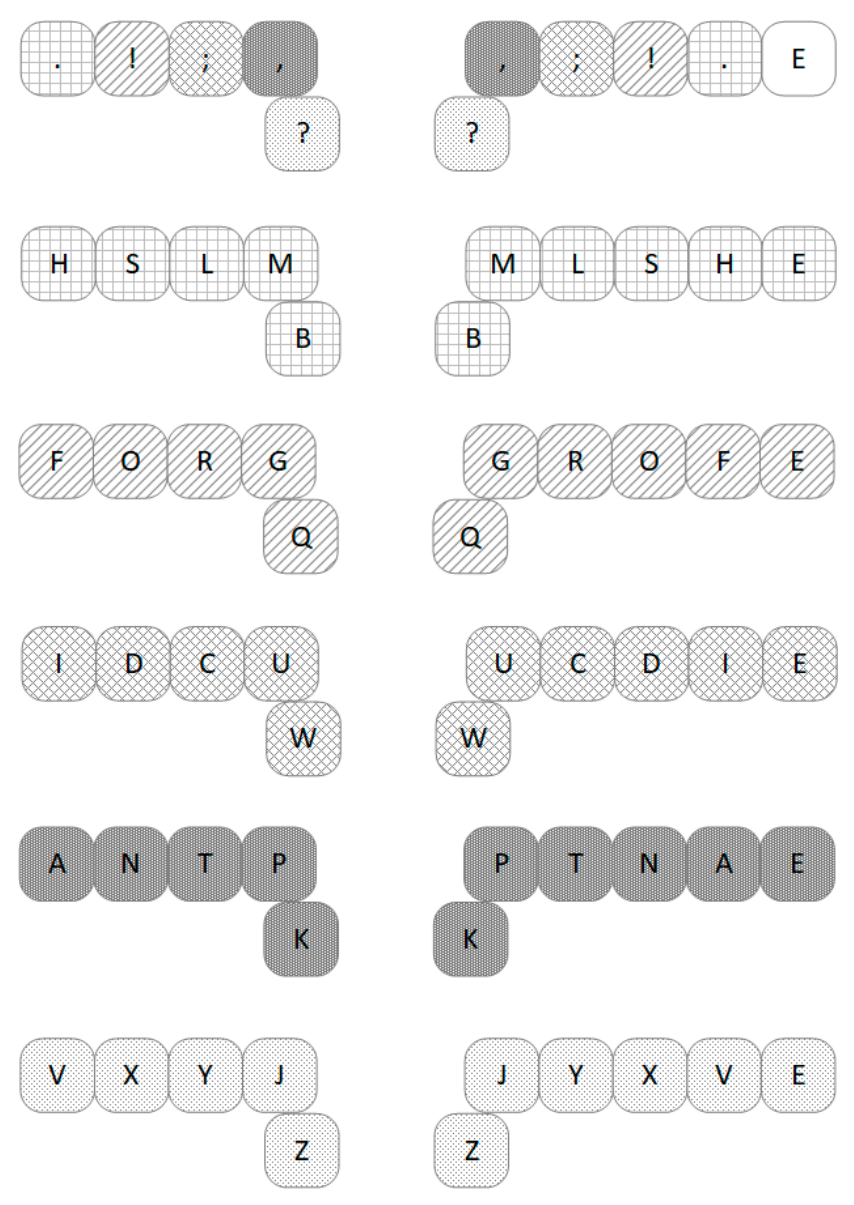

Figure 11. U-W-ORK+ arrangement.

The U-W-ORK+ arrangement, like the previous one, ensures almost evenly distributed loads to all typing fingers. In the case of the U-W-ORK+ arrangement, the difference between the most and least loaded fingers is $2 \%$. In addition, the index finger movement frequency decreased to $3.6 \%$. The greatest downside of the U-W-ORK+ arrangement is the big load to the right little finger, responsible for operating the key with the letter " $\mathrm{E}$ ".

Table 2 presents the values of the BI and DK metrics for the $\mathrm{W}-\mathrm{ORK}+$. It also shows the values of two additional metrics based on the function F-W-ORK+: load distribution to typing fingers (EL) and the movement frequency for the index finger while typing a single letter $(\mathrm{M})$. Both of the metrics proposed refer to typing a single character (special characters not included) requiring two keystrokes. The table shows the values of the metrics for a single keystroke (EL/2, M/2).

Table 2. Assessment of the W-ORK+ group arrangements.

\begin{tabular}{ccccc}
\hline & ABC & Q & MY & U \\
\hline BI & $100.00 \%$ & $100.00 \%$ & $100.00 \%$ & $100.00 \%$ \\
DK & $0.00 \%$ & $2.51 \%$ & $0.00 \%$ & $12.49 \%$ \\
$\mathrm{EL} / 2$ & $38.37 \%$ & $20.71 \%$ & $0.09 \%$ & $0.01 \%$ \\
$\mathrm{M} / 2$ & $19.30 \%$ & $18.20 \%$ & $4.46 \%$ & $3.56 \%$ \\
\hline
\end{tabular}

Only for U-W-ORK+ the DK ratio is more than $10 \%$. While it enables the frequency of the characters requiring the use of two keys to be reduced, it also puts a large load to the little finger of the right hand. All of the keyboards from the W-ORK+ group are fully alternating. It should also be emphasized that all W-ORK + keyboards are fully alternating for each language that contains the same letters as English. 
The indicator of even load to the fingers typing is by far the smallest in the case of optimized keyboards. In terms of the EL ratio, the worst is $\mathrm{ABC}-\mathrm{W}-\mathrm{ORK}+$, for which the ratio is twice as high as for the $\mathrm{Q}-\mathrm{W}-\mathrm{ORK}+$ arrangement. In terms of the movement of the index finger, the values obtained for the $\mathrm{ABC}-\mathrm{W}-\mathrm{ORK}+$ and $\mathrm{Q}-\mathrm{W}-\mathrm{ORK}+$ arrangements are similar, but differ significantly from the values obtained for the My-W-ORK+ and U-W-ORK+ arrangements.

\subsection{NUM-ORK+ Arrangement}

The last of the presented arrangement is the NUM-ORK+ keyboard. The NUM-ORK+ arrangement uses 11 keys, where all keys are dead keys. An additional key was assigned to the location of the default character. This enabled typing digits, the access to which is provided with the additional dead key. However, for the same reason, the keyboard is no longer $100 \%$ alternating. Some of the occurrences of the letter " $Z$ " will require using the same hand twice. This will take place when the left hand ends typing the letter preceding the letter " $Z$ ". Then, the left hand should be used again or the right hand should be used twice. The NUM-ORK+ arrangement presented in Figure 12 is developed on the basis of $\mathrm{My}-\mathrm{W}-\mathrm{ORK}+$.
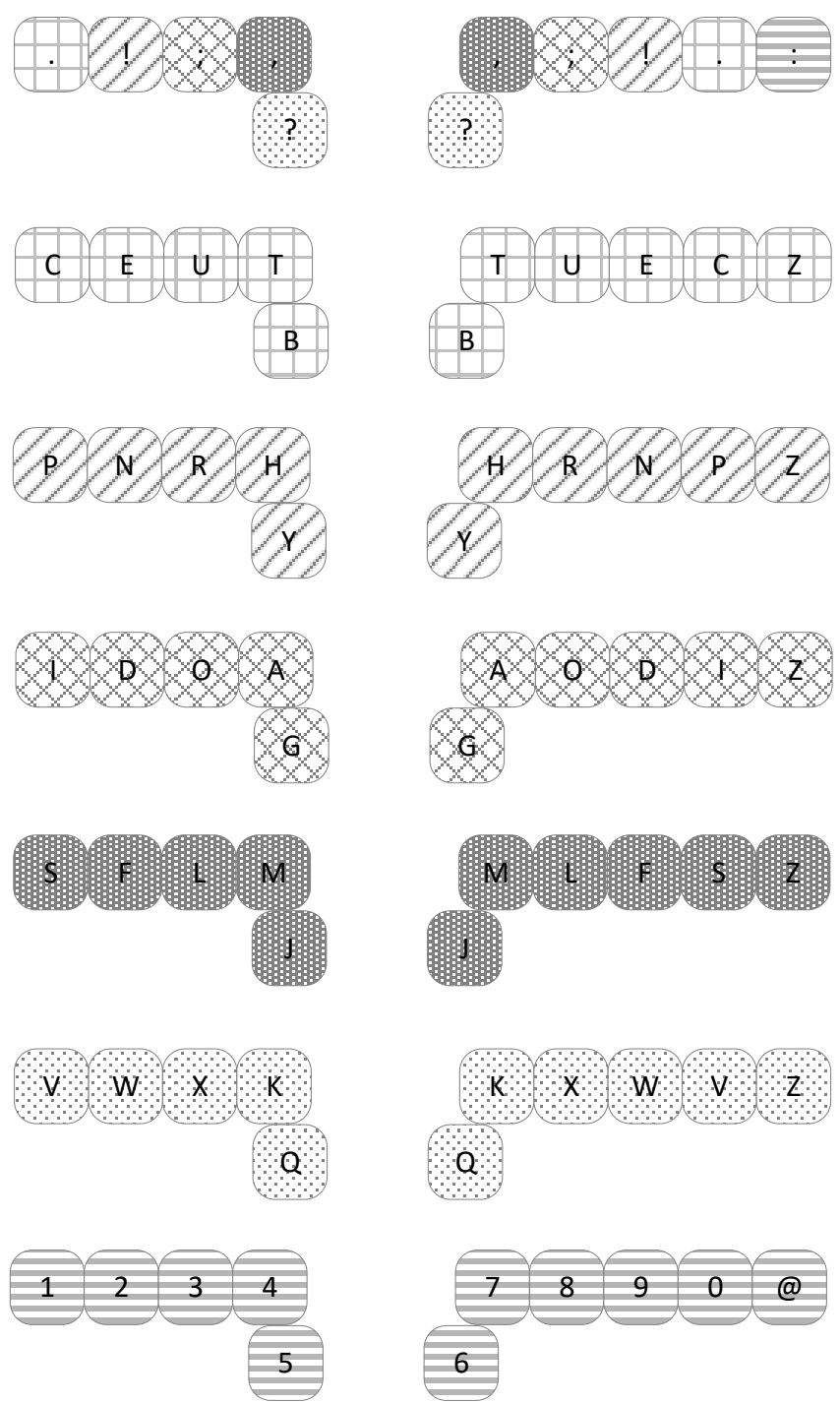

Figure 12. NUM-ORK+ arrangement. 
An unquestionable advantage of the NUM-ORK+ is supporting typing digits. However, with an additional dead key, the keyboard is no longer fully alternating. Additionally, accessing digits assigned to the right hand requires the same hand to be used repeatedly (twice).

\section{Discussion}

On the basis of the method for developing ORK keyboard, several examples of ORK+ keyboard layouts were created and presented in Section 3. The proposed layouts were divided into three groups, each of which was characterized by different assumptions and was aimed at showing various possibilities of using ORK layouts. Layouts of the first group (VX-ORK + ) use the fewest number of keys and have the lowest number of dead keys. Consequently, they can be considered the easiest to learn as they do not require a key combination to enter eight letters. Additionally, the $A$ and $Q$ layouts reflect the most popular keyboard layout-QWERTY. This aspect may additionally affect the ease of use of layouts by people who use the QWERTY layout on a daily basis. The last layout of this group ( $\mathrm{P}-\mathrm{VX}-\mathrm{ORK}+)$ has been optimized in order to maximize the frequency of using letters, the typing of which does not require the use of a key combination (DK), and also to maximize the alternation of typing (BI).

Layouts belonging to the second group ( $\mathrm{W}-\mathrm{ORK}+$ ) have a larger number of keys than the VX-ORK+ layouts. By increasing the number of keys and the number of dead keys, it was possible to achieve full alternation of typing $(B I=100 \%)$. At the same time, it caused the DK metric to drop to a very low level. In the U-W-ORK+ keyboard, for which this metric reaches the highest value ( $\mathrm{DK}=12.5 \%$ ), it is necessary to make frequent movements with the little finger (supporting the letter "E"). The example of the Q-W-ORK+ layout shows the possibility of adapting the QWERTY keyboard to a fully alternating layout. A fully alternating version of the alphabetic keyboard (ABC-W-ORK + ) was also proposed.

The last layout group presented (NUM-ORK+), consisting of 11 keys, all of which are dead keys, contains numerical characters. This arrangement is not fully alternating (some occurrences of the letter " $\mathrm{Z}$ " will require using the fingers of the same hand twice), but it contains as much as 11 characters more than the layouts of the W-ORK+ group. By adding another key, it would be possible to obtain the effect of full alternation also for this layout.

The presented examples of layouts differ in the number of keys, dead keys, as well as the criteria for arranging characters. As presented in the study, the results of individual evaluation criteria strongly depend on the layout parameters mentioned. According to the authors, comparing different layouts such as ORK or ORK+ makes sense only when the number of keys, dead keys, and characters to appear on the keyboard are equal. The requirements for the layout must also be similar, e.g., whether the layout should be as similar as possible to the most common QWERTY keyboard, or whether it should be optimized for selected criteria. Therefore, comparing the results for the groups of layouts presented in the study, could suggest incorrect conclusions regarding the individual layouts. All the presented layouts serve only to illustrate the wide possibilities of the ORK+ keyboards.

\section{Conclusions}

The idea behind the ORK type keyboards proposed by the authors is to decrease the number of the movements of the fingers when typing, through the development of keyboards with keys arranged in one row. In order to enable the use of the same keyboard on computers and mobile devices, the authors proposed the ORK+ solution. ORK+ keyboards consist of a smaller number of keys, as compared to conventional keyboards (e.g., those compliant with the ISO/IEC 9995-2:2009 standard [31]). The arrangements proposed reduce the number of finger movements, since only the index and little fingers operate two keys each, while the middle and ring fingers operate only one key each, which means that the vertical movements of these fingers are eliminated. The reduction of the number of 'character' keys has made it necessary to introduce dead keys, which in turn renders it difficult to access some letters. The difficulty consists in a need to use two keystrokes 
to type a single letter $(\mathrm{KSPC}=2)$. It is more difficult, and for the $\mathrm{W}-\mathrm{ORK}+$ arrangements impossible, to label each key with the characters it provides easy access to. The idea of using the key combination to enter a single character, presented in this study, can also be used for very small devices, such as smartwatches. The topic of small devices keyboards is the subject of many studies (e.g., [35-38]) and this topic still very current.

Therefore, ORK + arrangements require more detailed learning than as in the case of the QWERTY arrangement does, because typing some letters is more complicated. The downside can, however, prove to be an advantage of the ORKs+ arrangements, because the use of an ORK+ keyboard practically must be preceded by learning it. There is no such need in the case of the QWERTY keyboard. This, however, may contribute to less effective typing, such as typing with the index finger only or 'searching for letters' on the keyboard.

In addition to minimizing the number of finger movements, the $\mathrm{W}-\mathrm{ORK}+$ group keyboards have another important feature: they support fully alternating typing. This feature is commonly listed among most important optimization criteria for the arrangement of characters on the keyboard. Additionally, W-ORK + group keyboards are fully alternating for any language that contains the same letters as English.

ORK + also eliminates the need to use more than one row for writing. Thus, it implements the criterion of minimizing the frequency of using the upper and the lower rows of the keyboard for typing. In addition, movements across the home row are completely eliminated. In the case of the W-ORK+ group, the need to enter two consecutive characters using the same finger is also eliminated.

By adding one more key (to 12 keys), it would possible to add diacritic letters that are characteristic for a given language. This would create a universal (for any Latin-based language) keyboard, in which the location of all non-diacritical letters would be fixed.

ORK+ type keyboards are more difficult to use than the existing keyboards. They have, however, some major advantages, among which a decreased number of finger movements, and, in the case of the W-ORK+ arrangements, fully alternating typing have to be emphasized. Another important advantage is the significant reduction in the number of keys required on the keyboard, which enables the design of smaller keyboards. This is important in view of the fact that ORK+ keyboards are designed for the use also in small mobile devices.

Author Contributions: Conceptualization, R.P.; methodology, R.P.; software, R.P. and J.D.; validation, R.P. and P.Ł.; formal analysis, R.P., P.Ł. and J.D.; writing-original draft preparation, R.P., P.Ł. and J.D.; writing-review and editing, R.P. and J.D. All authors have read and agreed to the published version of the manuscript.

Funding: This study was conducted under a research project funded by a statutory grant of the AGH University of Science and Technology in Krakow for maintaining research potential.

Acknowledgments: We sincerely thank the anonymous reviewers and the editor for their carefulreading of our manuscript and their many insightful comments and suggestions.

Conflicts of Interest: The authors declare no conflict of interest.

\section{References}

1. Sholes, C.L. Improvement in Type-Writing Machines. U.S. Patent US207559A, 27 August 1878.

2. Dvorak, A.; Dealey, W. Typewriter Keyboard. U.S. Patent US2040248, 12 May 1936.

3. Heath, R.A.; Willcox, C.H. A stochastic model for inter-keypress times in a typing task. Acta Psychol. 1990, 75, 13-39. [CrossRef]

4. Işeri, A.; Ekşioğlu, M. Estimation of digraph costs for keyboard layout optimization. Int. J. Ind. Ergon. 2015, 48, 127-138. [CrossRef]

5. Wagner, M.O.; Yannou, B.; Kehl, S.; Feillet, D.; Eggers, J. Ergonomic modelling and optimization of the keyboard arrangement with an ant colony algorithm. J. Eng. Des. 2003, 14, 187-208. [CrossRef]

6. Liao, C.; Choe, P. Chinese keyboard layout design based on polyphone disambiguation and a genetic algorithm. Int. J. Hum. Comput. Interact. 2013, 29, 391-403. [CrossRef]

7. Pradeepmon, T.G.; Panicker, V.V.; Sridharan, R. Hybrid estimation of distribution algorithms for solving a keyboard layout problem. J. Ind. Prod. Eng. 2018, 35, 352-367. [CrossRef]

8. Anson, D.; George, S.; Galup, R.; Shea, B.; Vetter, R. Efficiency of the Chubon versus the QWERTY keyboard. Assist. Technol. 2001, 13, 40-45. [CrossRef] 
9. Smith, M.L.; Pickens, A.W.; Ahn, S.; Ory, M.G.; DeJoy, D.M.; Young, K.; Bishop, G.; Congleton, J.J. Typing performance and body discomfort among overweight and obese office workers: A pilot study of keyboard modification. Appl. Ergon. 2015, 46, 30-37. [CrossRef] [PubMed]

10. McLoone, H.E.; Jacobson, M.; Clark, P.; Opina, R.; Hegg, C.; Johnson, P. Design and evaluation of a curved computer keyboard. Ergonomics 2009, 52, 1529-1539. [CrossRef] [PubMed]

11. MacKenzie, I.S.; Zhang, S.X. The design and evaluation of a high-performance soft keyboard. In Proceedings of the CHI '99: The ACM Conference on Human Factors in Computing Systems, Pittsburgh, PA, USA, 15-20 May 1999; ACM: New York, NY, USA, 1999; pp. 25-31.

12. FITALY. One Finger Keyboard. 2006. Available online: http:/ /www.fitaly.com/fitaly / fitaly.htm (accessed on 2 November 2020).

13. Zhai, S.; Hunter, M.; Smith, B.A. The Metropolis Keyboard-An exploration of quantitative techniques for virtual keyboard design. In Proceedings of the UIST: The 13th Annual ACM Symposium on User Interface Software and Technology, San Diego, CA, USA, 6-8 November 2000; ACM: New York, NY, USA, 2000; pp. 119-128.

14. Zhai, S.; Hunter, M.; Smith, B.A. Performance optimization of stylus keyboards. Hum. Comput. Interact. 2002, 17, 89-129.

15. Hsiao, H.C.; Wu, F.G.; Chen, C.H. Design and evaluation of small, linear QWERTY keyboards. Appl. Ergon. 2014, 45, 655-662. [CrossRef] [PubMed]

16. Bi, X.; Smith, B.A.; Zhai, S. Multilingual touchscreen keyboard design and optimization. Hum. Comput. Interact. 2012, 27, 352-382.

17. Li, Y.; Chen, L.; Goonetilleke, R.S. A heuristic-based approach to optimize keyboard design for single-finger keying applications. Int. J. Ind. Ergon. 2006, 36, 695-704. [CrossRef]

18. Yin, P.Y.; Su, E.P. Cyber Swarm optimization for general keyboard arrangement problem. Int. J. Ind. Ergon. 2011, 41, 43-52. [CrossRef]

19. Kwon, S.; Lee, D.; Chung, M.K. Effect of key size and activation area on the performance of a regional error correction method in a touch-screen QWERTY keyboard. Int. J. Ind. Ergon. 2009, 39, 888-893. [CrossRef]

20. Park, Y.S.; Han, S.H. One-handed thumb interaction of mobile devices from the input accuracy perspective. Int. J. Ind. Ergon. 2010, 40, 746-756. [CrossRef]

21. Chang, J.; Choi, B.; Tjolleng, A.; Jung, K. Effects of button position on a soft keyboard: Muscle activity, touch time, and discomfort in two-thumb text entry. Appl. Ergon. 2017, 60, 282-292. [CrossRef] [PubMed]

22. Xiong, J.; Muraki, S. An ergonomics study of thumb movements on smartphone touch screen. Ergonomics 2014, 57, 943-955. [CrossRef] [PubMed]

23. Li, F.C.Y.; Guy, R.T.; Yatani, K.; Truong, K.N. The 1line keyboard: A QWERTY layout in a single line. In Proceedings of the 24th Annual ACM Symposium on User Interface Software and Technology, Santa Barbara, CA, USA, 16-19 October 2011; pp. 461-470.

24. Rakhmetulla, G.; Arif, A.S. Senorita: A Chorded Keyboard for Sighted, Low Vision, and Blind Mobile Users. In Proceedings of the 2020 CHI Conference on Human Factors in Computing Systems, Honolulu, HI, USA, 25-30 April 2020; pp. 1-13.

25. Wu, F.G.; Huang, Y.C.; Wu, M.L. New chording text entry methods combining physical and virtual buttons on a mobile phone. Appl. Ergon. 2014, 45, 825-832. [CrossRef] [PubMed]

26. Green, N.; Kruger, J.; Faldu, C.; St. Amant, R. A reduced QWERTY keyboard for mobile text entry. In Proceedings of the CHI'04 Extended Abstracts on Human Factors in Computing Systems, Vienna, Austria, 24-29 April 2004; pp. 1429-1432.

27. Bi, X.; Smith, B.A.; Zhai, S. Quasi-Qwerty Soft Keyboard Optimization. In Proceedings of the Proceedings of the 28th International Conference on Human Factors in Computing Systems-CHI '10, Atlanta, GA, USA, 10-15 April 2010; ACM Press: New York, NY, USA, 2010; p. 283.

28. Bi, X.; Zhai, S. IJQwerty: What Difference Does One Key Change Make? Gesture Typing Keyboard Optimization Bounded by One Key Position Change from Qwerty. In Proceedings of the 2016 CHI Conference on Human Factors in Computing Systems, San Jose, CA, USA, 7-12 May 2016; ACM: New York, NY, USA, 2016; pp. 49-58.

29. Dhakal, V.; Feit, A.M.; Kristensson, P.O.; Oulasvirta, A. Observations on Typing from 136 Million Keystrokes. In Proceedings of the 2018 CHI Conference on Human Factors in Computing Systems, Montreal, QC, Canada, 21-26 April 2018; ACM: New York, NY, USA, 2018; pp. 1-12.

30. Feit, A.M.; Weir, D.; Oulasvirta, A. How We Type: Movement Strategies and Performance in Everyday Typing. In Proceedings of the 2016 CHI Conference on Human Factors in Computing Systems, San Jose, CA, USA, 7-12 May 2016; ACM: New York, NY, USA, 2016; pp. 4262-4273.

31. ISO. ISO/IEC 9995-2:2009 Information Technology—Keyboard Layouts for Text and Office Systems—Part 2: Alphanumeric Section; ISO: Geneva, Switzerland, 2009.

32. Microsoft. Microsoft Keyboard Layout Creator. 2020. Available online: https://www.microsoft.com/en-us/download/details. aspx?id=102134 (accessed on 2 November 2020).

33. Norvig, P. English Letter Frequency Counts: Mayzner Revisited or Etaoin Srhldcu. 2012. Available online: http://norvig.com/ mayzner.html (accessed on 2 November 2020).

34. MacKenzie, I.S. KSPC (keystrokes per character) as a characteristic of text entry techniques. In Proceedings of the Mobile HumanComputer Interaction, 4th International Symposium, Mobile HCI, Pisa, Italy, 18-20 September 2002; Springer: Berlin/Heidelberg, Germany, 2002; pp. 195-210.

35. Komninos, A.; Dunlop, M. Text input on a smart watch. IEEE Pervasive Comput. 2014, 13, 50-58. [CrossRef]

36. Leiva, L.A.; Sahami, A.; Catala, A.; Henze, N.; Schmidt, A. Text entry on tiny qwerty soft keyboards. In Proceedings of the 33rd Annual ACM Conference on Human Factors in Computing Systems, Seoul, Korea, 18-23 April 2015; pp. 669-678. 
37. Qin, R.; Zhu, S.; Lin, Y.H.; Ko, Y.J.; Bi, X. Optimal-t9: An optimized t9-like keyboard for small touchscreen devices. In Proceedings of the 2018 ACM International Conference on Interactive Surfaces and Spaces, Tokyo, Japan, 25-28 November 2018; pp. 137-146.

38. Elazari, A.; Ovadia, E. Reduced Keyboard Disambiguating System and Method Thereof. U.S. Patent WO2017183035A1, 26 October 2020. 